\title{
A morphological and genetic analysis of conidiophore development in Neurospora crassa
}

\author{
Matthew L. Springer and Charles Yanofsky \\ Department of Biological Sciences, Stanford University, Stanford, California 94305 USA
}

\begin{abstract}
The filamentous fungus Neurospora crassa responds to nutrient deprivation and dessication by producing asexual spores, or conidia. These conidia are derived from differentiated aerial structures called conidiophores. The process of conidiation was analyzed in wild-type and morphological mutants using scanning electron microscopy (SEM) and specific fluorescent probes. The first discernible morphological step of conidiation is the transition from growth by hyphal tip elongation to growth by repeated apical budding, resulting in the formation of chains of proconidia that resemble beads on a string. The initial proconidial chains are morphologically distinct from those that form later and are capable of reverting to hyphal growth, whereas the later chains are committed to conidiation. As the proconidial chains are formed, nuclei migrate into the conidiophore, and cross-walls arise between adjoining proconidia in a series of steps that have been defined by staining with Calcofluor, a fluorescent chitin-binding probe. The chains ultimately disarticulate in several discrete stages into free, morphologically mature conidia. Different conidiation-defective mutants were shown to be blocked at distinct stages in conidiation. Our observations permit us to derive a developmental timeline of conidiation relating the occurrence of morphological changes and the stage blocked in specific mutants.
\end{abstract}

[Key Words: Conidiation; Neurospora; scanning electron microscopy; Calcofluor staining; developmental regulation]

Received January 4, 1989; revised version accepted February 15, 1989.

The asexual reproductive cycle of the filamentous fungus Neurospora crassa is well suited for the study of developmental gene regulation and differentiation (Turian and Bianchi 1972; Nelson et al. 1975; Schmidt and Brody 1976; Brody 1981). In the vegetative stage of its life cycle, Neurospora grows as filamentous hyphae that intertwine and fuse into a mycelial mat. The crosswalls that form throughout the hyphae have a central pore that allows cytoplasm and nuclei to stream freely from one 'cell' to its neighbors. In response to certain environmental cues, the fungus enters its asexual reproductive cycle and produces multinucleate spores, called macroconidia, on specialized aerial structures called conidiophores. (Macroconidia are morphologically and developmentally distinct from the smaller, uninucleate microconidia produced in old cultures. This paper is concerned mainly with macroconidia and will refer to them as conidia throughout.) The cues that induce conidiation are not well defined, but dessication, nutrient deprivation, carbon dioxide levels, and exposure to light all seem to play a role in the induction of aerial growth and subsequent conidiation (Sargent and Kaltenborn 1972; Nelson et al. 1975). Upon induction of conidiation, the actual initiation of conidiation is preceded by an aerial growth phase. Some of the resulting aerial hyphae generate chains of proconidia via a process of successive apical budding in which each apical bud gives rise to the next bud. Septation occurs between each pro- conidial element, and ultimately the chain disarticulates to release free conidia which, although held together by a thread-like connective, are easily dispersed by the slightest air currents (Turian and Bianchi 1972; Matsuyama et al. 1974). The conidia undergo a further biochemical maturation period of several days before they become dormant and capable of efficient germination (Schmidt and Brody 1976). Conidia germinate when they encounter a moist, nutritious substrate, giving rise to a new mycelium. It is possible to synchronize the process of conidiation by appropriate manipulation of a growing culture (Berlin and Yanofsky 1985a).

A brief scanning electron microscopic overview of conidiation has been provided by Seale (1973), and several studies have dealt with the morphological changes that occur during conidiation (Turian and Bianchi 1972; Nelson et al. 1975). Matsuyama et al. (1974), using light microscopy, proposed a simple scheme of conidiation involving five morphological steps: formation of specialized aerial branches, nuclear migration into the tips of these hyphae, swelling of the tips, apical budding of proconidial chains, and septation with subsequent separation of mature conidia. On the molecular level, it is known that the levels of certain proteins and mRNAs are elevated greatly during conidiation (Turian and $\mathrm{Ma}$ tikian 1966; Urey 1971; Berlin and Yanofsky 1985a). Several genes that are expressed more actively or only during conidiation have been isolated and characterized 
(Berlin and Yanofsky 1985b; Roberts et al. 1988; Roberts and Yanofsky 1989|. Similar studies have been performed on the morphologically more complex conidiation pathway in Aspergillus nidulans (Timberlake and Marshall 1988).

In this study we describe the sequence of events involved in wild-type conidiation using scanning electron microscopy (SEM) and specific fluorescent stains, and we correlate these events with analyses of expression of conidiation-specific genes (Berlin and Yanofsky 1985b). Also, we examine mutants that are defective in conidiation and identify their developmental blocks in the context of the steps we have defined in the process of conidiation.

\section{Results \\ Developmental sequence of conidiation}

The process of conidiophore development can be divided into early events, which consist of the formation of proconidial chains by repeated apical budding, and late events, which consist of septation between the proconidia and their subsequent separation into spores. Growth of the conidiophore ceases after the early events are completed.

The vegetative mycelium of $N$. crassa (Fig. 1a) is a tangled, fused mass of hyphae that grows through and over the substrate. Upon receiving the proper environmental signals, hyphae send out new branches oriented away from the substrate. These aerial hyphae grow and branch to form an aerial mass. Conidiation begins when some of these branches, destined to become conidiophores, change their growth mode from hyphal tip elongation to repeated apical budding. Budding begins about $2 \mathrm{hr}$ after conidiation is induced at $\sim 22^{\circ} \mathrm{C}$ under conditions described in Materials and methods. The initial few rounds of budding are distinctive in that they result in chains with interconidial junctions that are almost as large in diameter as the proconidia (Fig. 1b). These 'minor constrictions' are characteristic of the earliest parts of the proconidial chains and, hence, are referred to as minor constriction chains. At $4 \mathrm{hr}$ after induction, these chains make up the majority of the aerial mass. As the chains continue to bud, the newly formed interconidial junctions are smaller in diameter; we refer to these chains as major constriction chains. From this point on, all growth proceeds by the formation of major constriction chains, which make up almost the entire population of proconidial chains by $8 \mathrm{hr}$ after induction (Fig. 1c). Therefore, a typical proconidial chain consists of a short segment of minor constriction chain, followed by a much longer segment of major constriction chain. Proconidial chains also can branch as they grow by budding more than once from a single proconidium.

A distinguishing characteristic of minor constriction budding is the tendency to revert to hyphal growth. Figure 1d shows an aerial hypha that had begun minor constriction budding and then branched. The uppermost branch has reverted to hyphal growth. Growth of a single chain can fluctuate repeatedly between minor constriction budding and hyphal tip elongation. However, major constriction chains have never been observed to revert to hyphal growth or minor constriction budding. The two types of chains differ also in that minor constriction chains sometimes are found in several day-old submerged liquid cultures, whereas major constriction chains are not.

Cross-walls, or septa, begin to form in the interconidial junctions $8-10 \mathrm{hr}$ after induction and are visible by SEM as ridges or collars between adjacent proconidia (Fig. le). Subsequently, the septa undergo thickening, followed by furrow formation and the separation of adjacent conidia, which remain held together by a connective (Fig. lf). The first connectives are seen $10 \mathrm{hr}$ after induction; by $16 \mathrm{hr}$, the vast majority of conidia have separated from one another (Fig. lg). The transition from the early events to the late events appears to take place between 8 and $10 \mathrm{hr}$, a period during which both septation and separation begin.

Most of the conidia in a culture are produced by successive apical budding, as described above, a process called holoblastic development (Cole 1986); therefore, these conidia are referred to as blastoconidia. However, a small number of conidia-like elements arise in a different manner. During or after septation of proconidial chains, extra cross-walls appear in the parent hypha that gave rise to the conidiophore. The distance between the extra cross-walls nearest the conidiophore is $\sim 10 \mu \mathrm{m}$, compared with an average distance between normal hyphal cross-walls of $\sim 50 \mu \mathrm{m}$. The interseptal distance increases with increasing distance from the chain. The cross-walls undergo the same thickening and separation as those in the conidiophore, resulting in the disarticulation of hyphal segments ranging in size from 5 to $30 \mu \mathrm{m}$, depending on the distance from the conidiophore. This is characteristic of thallic-arthric development /Cole 1986), and so these disarticulated hyphal segments are referred to as arthroconidia. Arthroconidia first appear $\sim 10-12 \mathrm{hr}$ postinduction and are prevalent throughout the culture by $20 \mathrm{hr}$, comprising roughly $1 \%$ of the total conidial mass (Fig. 1h). The arthroconidia can be distinguished from the oblong or round blastoconidia by their cylindrical shape.

More detailed views of septation and conidial separation are presented in Figure 2. Figure 2a shows an interconidial junction before septation has occurred. In Figure $2 b$, a cross-wall has formed, which is visible in this SEM preparation as a collar, owing to slight shrinkage of the sample during preparation. In Figure $2 c$, the cross-wall has thickened, and a furrow has formed. (Had this sample also shrunk, the cross-wall would have appeared as two collars with a furrow between them.) Figure $2 \mathrm{~d}$ shows a similar sample in which the conidia have begun to separate. The connective initially is short and thick but, ultimately, is drawn out into a longer, thin thread of material (Fig. 2e).

Fluorescent probes have been used to characterize further the steps involved in conidiation. The stains used are Calcofluor, which stains chitin and other $\beta$-linked polysaccharides (Darken 1961; Maeda and Ishida 1967), and the DNA stain Hoechst 33258. Chitin is a major 

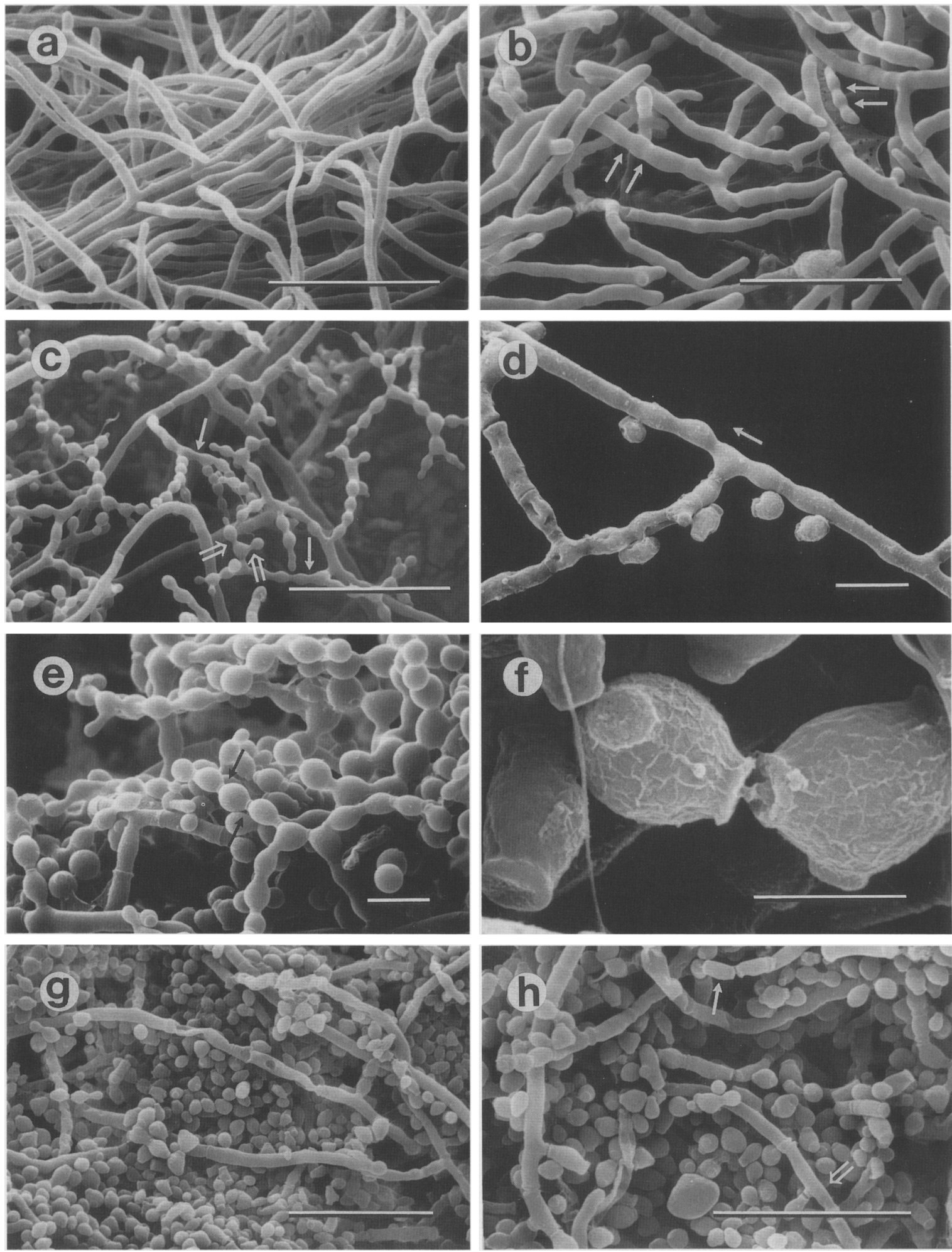

Figure 1. Scanning electron micrographs of developing wild-type conidiophores. $(a)$ Vegetative hyphae. $(b)$ Minor constriction chains. Arrows point to constrictions. (c) Major constriction chains. Double-line arrows point to major constrictions; small arrows point to minor constrictions. (d) Minor constriction chain reverting to hyphal growth. Arrow denotes direction of growth. (e) Conidiophore with cross-walls (arrows). $(f)$ Connective between two mature conidia. $(g)$ Culture after separation of conidiophores. $(h)$ Culture with arthroconidia ranging in size from small arthroconidia (small arrows) to longer arthroconidial logs (double-line arrows). Hours after induction of conidiation and scale bar lengths; $(a) 0 \mathrm{hr}, 50 \mu \mathrm{m} ;(b) 4 \mathrm{hr}, 50 \mu \mathrm{m} ;(c) 8 \mathrm{hr}, 50 \mu \mathrm{m} ;(d) 4-8 \mathrm{hr}, 10 \mu \mathrm{m} ;(e) 12 \mathrm{hr}, 10 \mu \mathrm{m} ;(f)$ $16 \mathrm{hr}, 5 \mu \mathrm{m}$; (g) $16 \mathrm{hr}, 50 \mu \mathrm{m}_{i}(\mathrm{~h}) 24 \mathrm{hr}, 50 \mu \mathrm{m}$. 

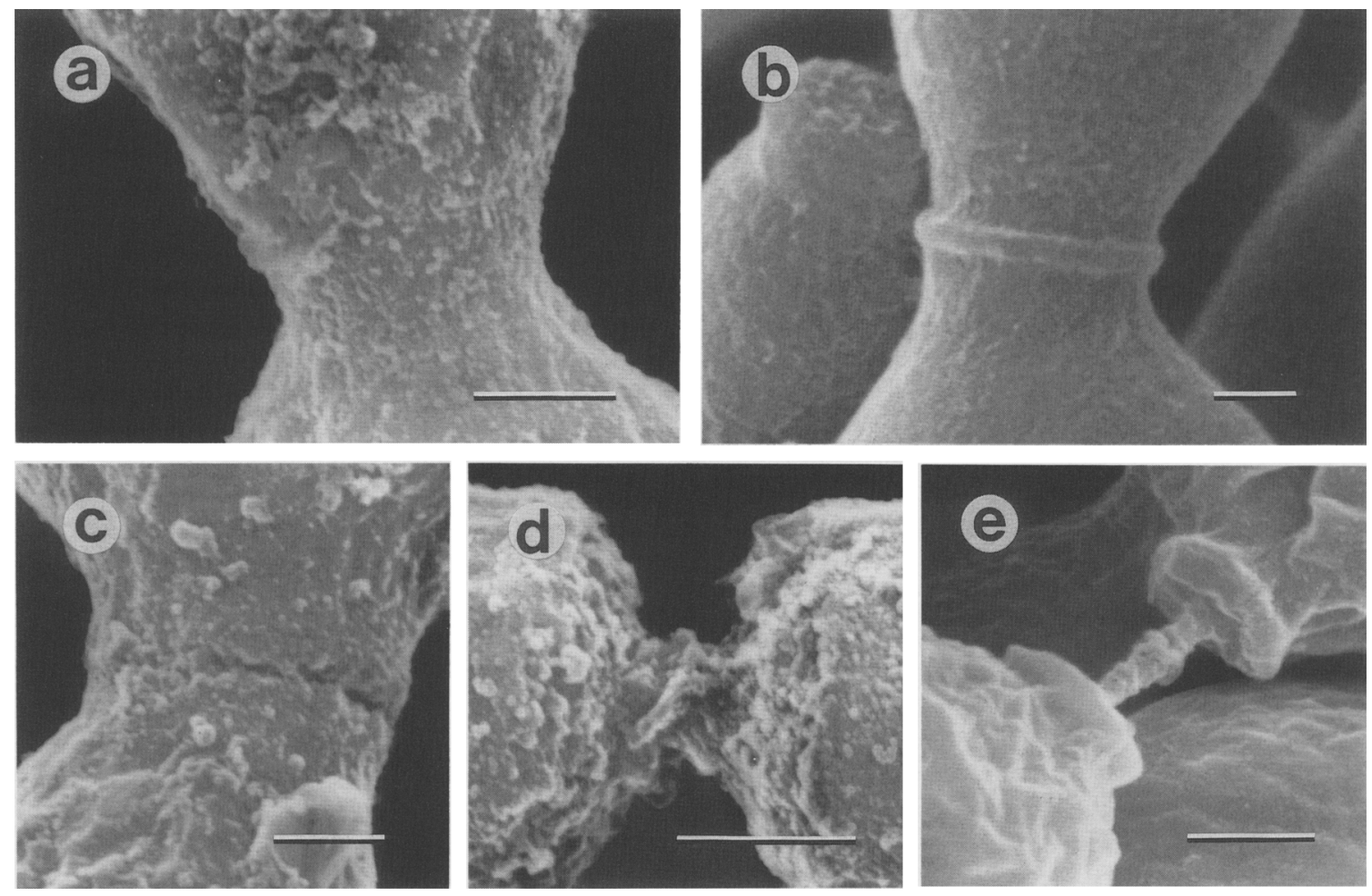

Figure 2. SEM of septation and separation. Bars, $1 \mu \mathrm{m}$. $(a)$ Interconidial junction before septation; $(b)$ collar; $(c)$ furrow formation; $|d\rangle$ beginning of connective formation; $(e)$ finished connective.

component of the cross-wall (Hunsley and Gooday 1974), so Calcofluor outlines the pattern of septation during conidiation. The outer cell wall contains chitin in smaller amounts as well (Mahadevan and Tatum 1965) but does not appear nearly as bright as the crosswalls, which are viewed edge on. Hoechst staining reveals the distribution of nuclei during chain growth. Vegetative hypae stained with Calcofluor and Hoechst are shown in Figure 3, a and b, respectively.

By combining Hoechst fluorescence with dark-field microscopy, we can observe the distribution of nuclei throughout minor and major constriction chains. Darkfield optics reveal the outline of the chain so that the nuclei can be viewed in the correct context (Fig. 3c). The nuclei appear to migrate into the conidiophore as it is being formed, but they usually do not enter a given proconidial bud until it has finished growing. This may be a property unique to proconidial growth, or it may be related to the general exclusion of nuclei from the first few micrometers of hyphal tips (Matsuyama et al. 1974).

Calcofluor staining allows us to examine the relation of budding growth and septation. If a young proconidial chain is stained with Calcofluor, no cross-walls are seen (Fig. 3d). Likewise, we rarely observe chains with cross walls both present in their older, proximal regions and absent in their newer, distal regions. Taken together, these results suggest that septation of a particular chain occurs only after chain growth is completed. Calcofluor staining also confirms the suggestion by Matsuyama et al. (1974) that individual cross-walls are laid down in a random order through the conidiophore, as opposed to septation beginning at one end and proceeding sequentially down the chain (Fig. 3e).

Calcofluor also has been used to observe the structural changes accompanying conidial separation. When an interconidial junction has reached the furrow stage, Calcofluor staining reveals two thick bands of material, one on each side of the furrow (Fig. 3f). Higher magnification shows that each band is made up of two thinner bands, yielding a Calcofluor staining pattern of a doublet of doublets. This 'double-doublet' pattern is shown in Figure $3 \mathrm{~g}$. As the conidia separate and the connective forms, the stained material that makes up the two inner bands appears to condense, changing from two thin plates to thick cylinders of smaller diameter. The cylinders ultimately are drawn out into the long, thin connective thread (Fig. $3 \mathrm{~h}$ ). The outer bands remain unchanged and become the bud scars of the two conidia. This suggests that the connective is derived from the material that formed the two inner bands. There is also a small button of material in the middle of the finished connective that is visible by both Calcofluor staining and SEM; this material may be derived from the plug that fills the interconidial septal pore before separation.

\section{Conidiation mutants}

To determine the stages of conidiation that are blocked in different mutant strains, we have examined wellknown Neurospora mutants that are either aconidial or 

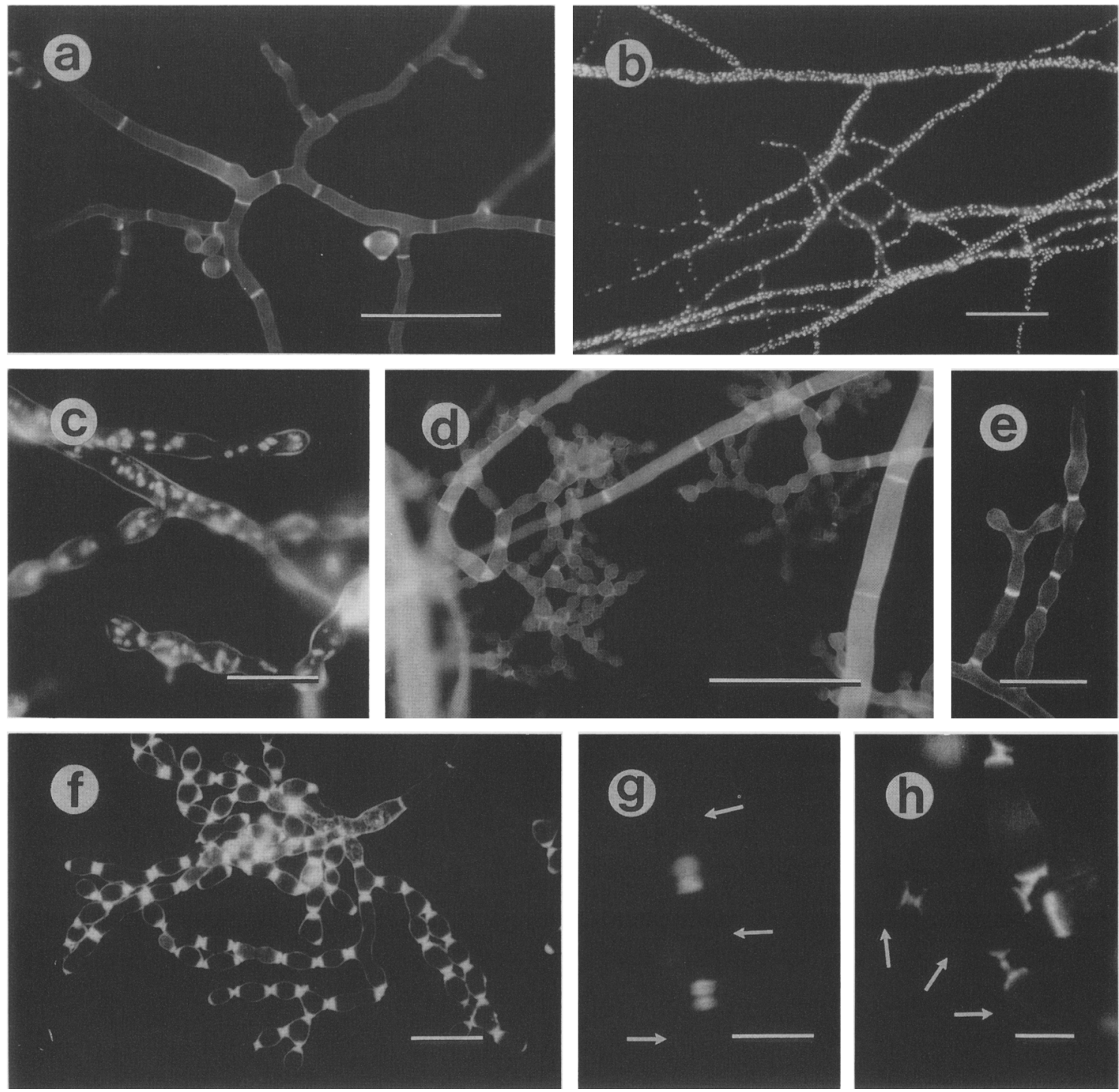

Figure 3. (a) Hyphal cross-walls visualized with Calcofluor. (b) Multinucleate hyphae stained with Hoechst 33258. (c) Combined dark-field and Hoeschst 33258 fluorescence images of nuclei that are moving through proconidial chains. (d) Proconidial chain stained with Calcofluor before septation. Some cross-walls are evident in the hyphae. (e) First interconidial cross-walls appearing in random order, visualized with Calcofluor. $(f)$ Combined dark-field and Calcofluor fluorescence images of a conidiophore with thickened cross-walls (double-doublet stage). $(g)$ Close-up view of a double-doublet, stained with Calcofluor. Arrows point to conidia. $(h)$ Connectives visualized with Calcofluor. Arrows point to conidia that are not visible in the photograph. Scale bars: $\{a \mid 50 \mu \mathrm{m} ;(b) 50 \mu \mathrm{m} ;(c)$ $20 \mu \mathrm{m}_{;}(d) 50 \mu \mathrm{m}_{\text {; }}(e) 20 \mu \mathrm{m}_{\text {; }}(f) 20 \mu \mathrm{m}_{;}(g) 5 \mu \mathrm{m}_{;}(h) 5 \mu \mathrm{m}$.

defective in some aspect of conidiation (for a list, see Table 1). In two of these mutants, aconidiate-2 (acon-2) and fluffyoid $(f l d)$, conidiation is blocked before the minor constriction stage, i.e., they produce a normal complement of aerial hyphae that never bud. acon-2, which is temperature sensitive (Matsuyama et al. 1974), is aconidial at $34^{\circ} \mathrm{C}$ (Fig. 4a) but conidiates at $25^{\circ} \mathrm{C}$. Conidiophores made at the permissive temperature appear normal by SEM and fluorescence analysis, although the timing of their appearance is delayed relative to that in wild type. acon-2 also can be induced to make morphologically normal conidia at the nonpermissive temperature if grown under carbon-limiting conditions. Occa- sionally the fld mutant produces flecks of conidia but is generally aconidial (Fig. 4b). However, it can be induced to conidiate by growing it under carbon-limiting conditions at $25^{\circ} \mathrm{C}$. Conidiation is uninducible at $34^{\circ} \mathrm{C}$. This apparent temperature dependency is different from that of $a c o n-2$ in that severe nutrient deprivation causes conidiation in fld only at the permissive temperature, whereas acon-2 conidiates at the permissive temperature under normal nutrient conditions and partial starvation for carbon forces conidiation at the nonpermissive temperature.

Two other mutants, aconidiate-3 (acon-3) and fluffy (f1) (Matsuyama et al. 1974), are blocked before the major 
Table 1. Mutants analyzed where 74-OR23-1A was used as wild type

\begin{tabular}{|c|c|c|}
\hline Genotypes & $\begin{array}{l}\text { Allele } \\
\text { number }\end{array}$ & Comments \\
\hline aconidiate-2 (acon-2) & RS91t & $\begin{array}{l}\text { no minor constriction } \\
\text { budding }\end{array}$ \\
\hline aconidiate- 3 (acon-3) & RS503 & $\begin{array}{l}\text { no major constriction } \\
\text { budding }\end{array}$ \\
\hline adherent $(a d h)$ & NM227 & $\begin{array}{l}\text { few blastoconidia; makes } \\
\text { arthroconidia }\end{array}$ \\
\hline carpet $(c p t)$ & P564 & $\begin{array}{l}\text { aconidial with short } \\
\text { aerial hyphae; } \\
\text { produces some conidia } \\
\text { when dessicated }\end{array}$ \\
\hline $\begin{array}{l}\text { conidial-separation-1 } \\
(c s p-1)\end{array}$ & UCLA37 & conidia do not separate \\
\hline $\begin{array}{l}\text { conidial-separation- } 2 \\
\text { (cps-2) }\end{array}$ & UCLA101 & $\begin{array}{l}\text { conidia do not separate, } \\
\text { appears to be blocked } \\
\text { at double-doublet stage }\end{array}$ \\
\hline crisp-1 $|c r-1\rangle$ & B123 & $\begin{array}{l}\text { abundant but normal } \\
\text { conidiophores on very } \\
\text { short aerial hyphae }\end{array}$ \\
\hline curly (cy) & $\mathrm{Cl} 170 \mathrm{c}$ & conidia do not separate \\
\hline dapple $(d a)$ & $\mathrm{R} 2526$ & $\begin{array}{l}\text { conidia separate too } \\
\text { easily }\end{array}$ \\
\hline easily wettable $\{e a s\}$ & UCLA191 & $\begin{array}{l}\text { conidia lack hydrophobic } \\
\text { rodlet layer }\end{array}$ \\
\hline fluffy $(f l)$ & $\mathrm{P}$ & $\begin{array}{l}\text { no major constriction } \\
\text { budding }\end{array}$ \\
\hline fluffyish $(f l s)$ & STL6 & $\begin{array}{l}\text { conidiation delayed, slow } \\
\text { vegetative growth }\end{array}$ \\
\hline fluffyoid $(f 1 d)$ & P628 & $\begin{array}{l}\text { no minor constriction } \\
\text { budding }\end{array}$ \\
\hline frost $(f r)$ & B110 & $\begin{array}{l}\text { no blastoconidia; makes } \\
\text { arthroconidia }\end{array}$ \\
\hline granular (gran) & B42 & $\begin{array}{l}\text { exaggerated major and } \\
\text { minor constrictions }\end{array}$ \\
\hline mat (mat) & B57 & $\begin{array}{l}\text { conidia show abnormal } \\
\text { morphology }\end{array}$ \\
\hline $\begin{array}{l}\text { morphological-2 } \\
\text { (mo-2) }\end{array}$ & R2464 & $\begin{array}{l}\text { conidia show abnormal } \\
\text { morphology }\end{array}$ \\
\hline pile (pi) & $\mathrm{B} 101$ & $\begin{array}{l}\text { few major constriction } \\
\text { chains; slow growing } \\
\text { mycelium }\end{array}$ \\
\hline scumbo $(s c)$ & 5801 & abnormal conidiophores \\
\hline skin $(s k)$ & B106 & $\begin{array}{l}\text { slow growth, no aerial } \\
\text { hyphae }\end{array}$ \\
\hline sponge (spg) & none & $\begin{array}{l}\text { few major constriction } \\
\text { chains; slow growing } \\
\text { mycelium }\end{array}$ \\
\hline tangerine $($ tng $)$ & $\mathrm{p} 4474$ & $\begin{array}{l}\text { has giant proconidia that } \\
\text { engulf neighboring } \\
\text { conidia and hyphae }\end{array}$ \\
\hline
\end{tabular}

a See Perkins et al. (1982) for references.

constriction stage, acon-3 makes minor constriction chains that appear normal (Fig. 4c) and frequently revert to hyphal growth. The interconidial junctions of these minor constriction chains can form septa, but these cross-walls do not proceed to thicken and separate as in wild type (data not shown). Some chains, more closely resembling major constriction chains, are present in cultures that are slightly dessicated. The fl mutant (Fig. 4d) does not develop as far as acon-3; typically, conidio- phores bud a few times and then stop growing, and interconidial cross-walls are not formed.

Other mutants that are blocked before the major constriction stage, such as sponge (spg) and pile (pi), are defective in mycelial growth as well as aerial growth; therefore, their defects are not conidiation specific (data not shown). The mutant carpet (cpt) grows slowly over the agar, forming short aerial hyphae but no conidiophores; however, if $c p t$ cultures become dessicated, conidiophores are produced that appear normal on the microscopic level (data not shown).

Some mutants that produce abnormal conidia, such as granular (gran), are not actually blocked during development; rather, some of the steps in conidiation proceed aberrantly. During the minor constriction stage, the interconidial junctions of gran are thicker and more amorphous than they are in wild type (Fig. 4e). During the later major constriction stage, however, the junctions are unusually small and numerous (Fig. 4f). An individual wild-type proconidium typically gives rise to between 1 and 3 buds, whereas major constriction proconidia in gran frequently produce $5-10$ buds. Calcofluor staining reveals that in gran conidiophores, septation and separation involve all the normal intermediates, such as double-doublets. The mature conidia are unusually round compared with wild type, and the excessive budding results in a multitude of bud scars that stain with Calcofluor.

The tangerine (tng) mutant deviates from the normal developmental process during bud growth. Whereas wild-type proconidia grow until they reach a particular size (typically 5-10 $\mu \mathrm{m}$ ) and then cease growing, a small percentage of $t n g$ proconidia continue to grow to sizes of $100 \mu \mathrm{m}$ or more. Eventually some reach macroscopic proportions. Figure $4 \mathrm{~g}$ shows two moderately sized examples; larger conidia were fragile and did not withstand preparation for the SEM. These giant conidia engulf other conidia or hyphae while they are growing. Examination of thin sections of giant conidia under transmission EM reveal that they lack cell walls (data not shown). Whether the lack of a cell wall is a cause or a result of the primary tng defect is not known.

Three mutants, conidial-separation-1 (csp-1), conidialseparation-2 (csp-2) (Selitrennikoff et al. 1974), and curly (cy) (D. Perkins, unpubl.), are developmentally normal, with the exception that conidia fail to separate. When slant cultures of these mutants are tapped, a procedure that generally releases copious amounts of conidia from wild type, no conidia are freed. When stained with $\mathrm{Cal}-$ cofluor, csp-2 conidiophores show the double-doublet pattern at all interconidial junctions (Fig. 4h), but there is never evidence of connective formation. This suggests that csp-2 is blocked at the double-doublet stage, after the septum has thickened fully but before its conversion into the connective. This is consistent with the observation of Selitrennikoff et al. (1974) that the csp mutants lack the cell-wall autolytic activity normally associated with conidiation. Mutants csp-1 and cy both show highly variable Calcofluor staining patterns in that some interconidial junctions are at the double-doublet stage, some make connectives, and some never lay down septa. 

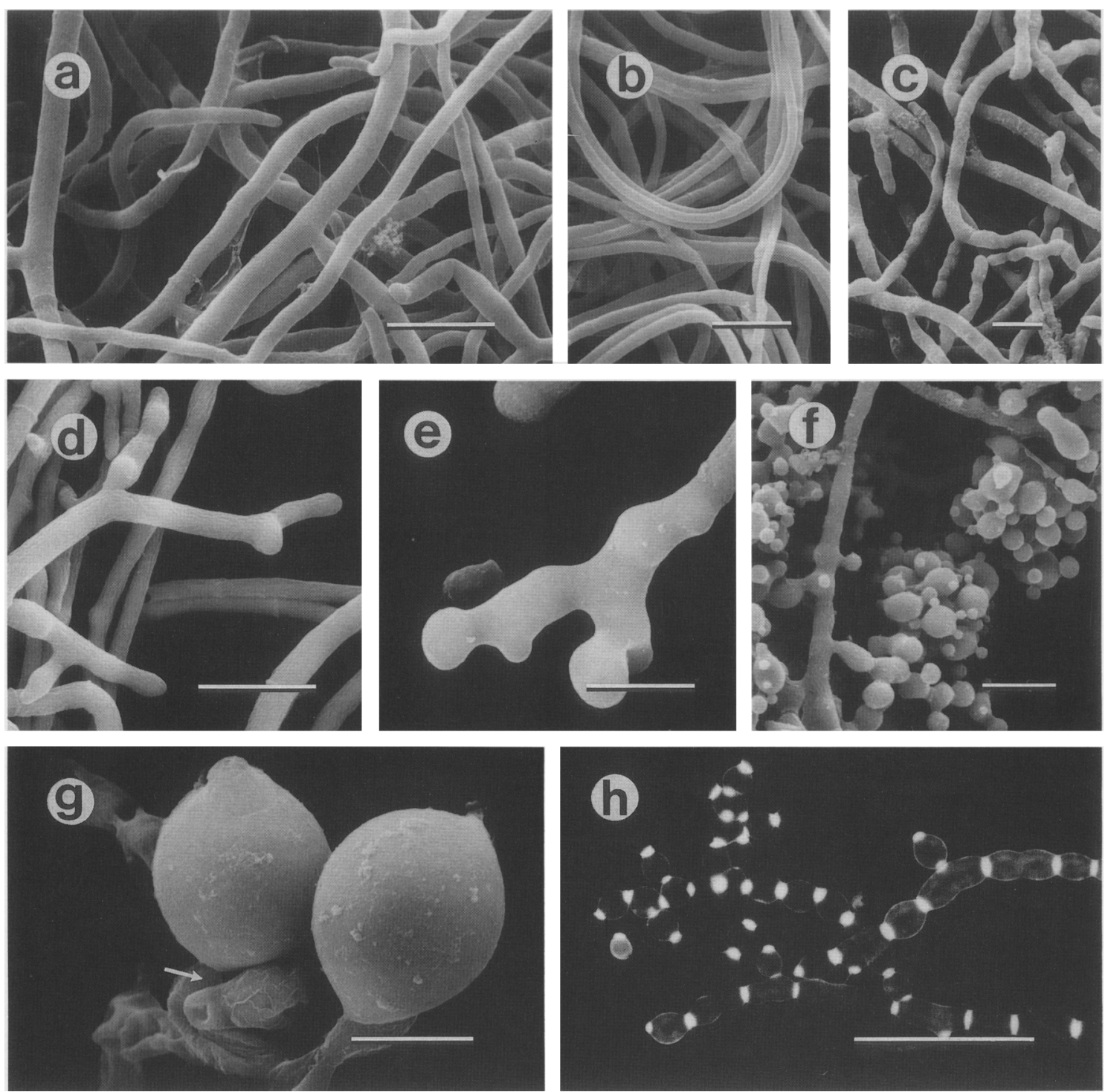

Figure 4. Conidiation mutants. All are visualized with SEM except $h$, which is visualized by Calcofluor staining and fluorescence. $(a)$ acon-2 at its nonpermissive temperature; $(b) f l d ;(c)$ acon-3; $(d) f l_{;}(e)$ gran early budding; $(f)$ gran later budding; $(g)$ tng giant conidia. Arrow points to normal-sized conidium. $(h) c s p-2$ conidiophore, stained with Calcofluor. Scale bars: $(a-d) 20 \mu \mathrm{m}_{;}(e) 10 \mu \mathrm{m} ;(f) 20 \mu \mathrm{m}$; (g) $10 \mu \mathrm{m} ;(h) 50 \mu \mathrm{m}$.

The mutant dapple $(d a)$ has the opposite phenotype; da conidiophores break apart without being disturbed. For this reason, further analysis of this mutant was not undertaken.

Other mutants examined include easily wettable (eas) and crisp-1 (cr-1). The mutant eas is so named because of the easily wettable nature of its conidia, as opposed to the water repellency that is characteristic of wild-type conidia (Selitrennikoff 1976). eas conidia appear to lack the hydrophobic protein coat called the rodlet layer (Beever and Dempsey 1978). The cr-1 mutant exhibits precocious conidiation and is deficient in adenylate cyclase activity (Terenzi et al. 1974). Both mutants produce conidiophores that appear normal when examined by SEM and with fluorescent probes (data not shown). Additional morphological mutants that were examined are described in Table 1.

\section{Ordering mutant blocks}

The phenotypes of some of the morphological mutant strains suggest that their gene products may act in the following order: acon-2 and fld, acon-3 and fl, gran, csp-1, and csp-2. To order mutational blocks more accurately, we constructed the following double mutants isolated from nonparental diatype unordered asci (Perkins 1966; Davis and de Serres 1970): acon-2; acon-3, fl; acon-2, fl; acon-3, acon-2;fld, and fl;fld. The cross of the strains containing the linked genes, acon-3 and fld, proved to be more difficult in terms of both viability of perithecia and scoring of progeny; thus, we did not pursue it further.

Double mutants acon-2;acon-3 and fl;acon-2 exhibited the acon- 2 mutant phenotype at $34^{\circ} \mathrm{C}$, i.e., they never formed minor constriction chains. At $25^{\circ} \mathrm{C}$, the 
permissive temperature for acon-2, these double mutants made minor constriction chains and did not continue to the major constriction stage, thus exhibiting the phenotypes of the acon-3 and $f l$ single mutants. Similarly, the fl;fld double mutant exhibited the fld phenotype. These results are consistent with the morphological observations, suggesting that the acon-2 gene product exerts its influence on conidiation before the acon -3 and $f l$ products do and that the fld gene product acts before that of the $f l$ gene.

The double mutant containing acon- 3 and $f 1$ mutations, both of which appear to affect the same stage of development, made some minor constriction chains and no major constriction chains, resembling both single mutants phenotypically. However, the minor constriction chains were rarer in the double mutant than in either single mutant. Similarly, acon-2;fld did not make proconidial chains of any sort at $34^{\circ} \mathrm{C}$.

\section{A decrease in blastoconidiation is correlated with an increase in arthroconidiation}

Two of the mutations we examined drastically increase the production of arthroconidia. In both mutants, the blastoconidiophores produced are either unusually short or nonexistent. The mutant adherent (adh) makes very short blastoconidiophores (Fig. 5a), which develop through the usual intermediate stages described above. This mutant also produces many more arthroconidia than does wild type (Fig. 5b). When stained with Calcofluor, an atypical diffuse fluorescent band bisects the arthroconidia (Fig. 5c), suggesting that extra chitin is being assembled. This diffuse band is never seen in wild type.

The mutant frost $(f r)$, which is defective in glucose-6phosphate dehydrogenase (Scott 1976), forms a contorted aerial mass that resembles coral (Fig. 5d), rather than forming normal aerial hyphae. An occasional long, straight aerial hypha bears small clumps of more corallike hyphae, which form cross-walls and separate in 1-day-old agar spot cultures (see Materials and methods). Much of the aerial mass disarticulates into twisted and bent arthrococonidia by the second day of growth. Using Calcofluor staining, we observe connectives between these arthroconidia, but not double-doublets (Fig. 5e). In both $a d h$ and $f r$, reduction in the number of blastoconidiophores is associated with overproduction of arthroconidia.

\section{Discussion}

\section{Conidiation follows a developmental program}

The relative simplicity of conidial development in Neurospora, along with the wealth of mutants defective in this process (Perkins et al. 1982), makes conidiation in Neurospora an attractive subject for the study of developmental gene regulation. Conidiophore development appears to involve a series of discrete morphological stages. The simplest hypothesis to explain conidiation is that it follows a built-in developmental program triggered by nutrient deprivation, undoubtedly involving sequential gene expression. In this study we have used
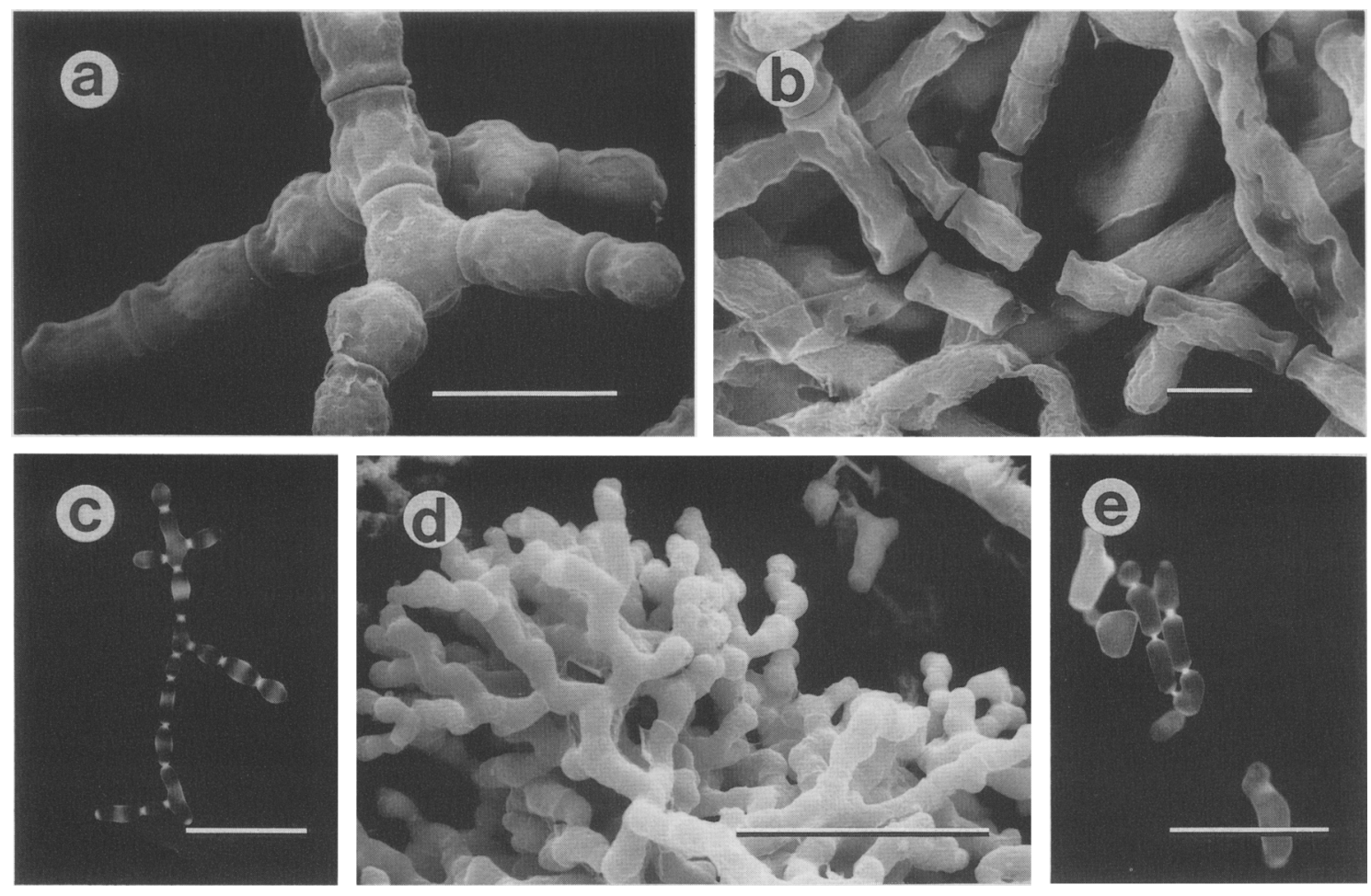

Figure 5. Effects of $a d h$ and $f r$ mutations on conidiation. (a) Scanning electron micrograph of adh blastoconidiophore after septation; (b) $a d h$ arthroconidia viewed by SEM; $(c)$ diffuse staining bands bisecting adh arthroconidia viewed by Calcofluor fluorescence; $(d) f r$ aerial hyphae, viewed with SEM; $(e)$ fr arthroconidia stained with Calcofluor. Scale bars: $\{a, b) 10 \mu \mathrm{m}_{;}(c-e) 50 \mu \mathrm{m}$. 
SEM and specific fluorescent probes to examine the sequence of morphological changes that occur during conidiation, and to determine at which step this process is blocked in specific mutants. The phenotypes of the morphological mutants suggest they are blocked at specific steps in conidial development and that the products of the wild-type alleles of these genes participate in the completion of these steps. Our interpretation of the developmental sequence of events in conidiation is summarized in Figure 6 as a developmental time line relating time, morphological changes, and mutational blocks.

The prelude to conidiation is the production of aerial hyphae. No mutant is known that exhibits normal vegetative growth but is unable to make aerial hyphae. The mutant skin $(s k)$ is defective in aerial growth; however, its vegetative growth also is impaired somewhat (see Table 11.

The first step in the conidiation process is the formation of minor constriction chains. This step is blocked in mutants acon-2 and fld, both of which can be induced to conidiate under certain starvation conditions. These mutant alleles could encode partially defective proteins, which accumulate to levels high enough to allow conidiation under starvation conditions. Alternatively, the gene products may sense nutrient levels and switch on conidiation when a critical level is reached; in this case, the mutant proteins would require conditions of greater nutritional stress to trigger conidiation.
As wild-type proconidial chains grow, they undergo several rounds of minor constriction budding and then switch to major constriction budding for the duration of growth. This switch signifies the commitment to budding growth, in that minor constriction chains revert frequently to hyphal growth, whereas major constriction chains do not. The mutants acon-3 and $f 1$ are blocked before the major constriction stage. fl makes some short minor constriction chains and then aborts growth. acon-3 continues to grow by minor constriction budding, and occasionally reverts to hyphal growth.

The conidial budding process can be considered as a cycle of steps that occurs each time a bud is formed. Presumably, the first step involves the organization of growth from a particular site on the surface of a previously formed conidium; this region ultimately becomes the interconidial junction and forms a cross wall. The mutant gran appears to be altered at this stage, during minor constriction growth, the interconidial junctions are amorphous and larger than those in wild type, and during major constriction growth, the junctions are unusually well defined and numerous. Subsequent budding steps in the cycle include radial growth of the proconidium, cessation of growth when the bud reaches a critical size, and the migration of nuclei into the proconidium, after which the cycle starts again with the growth of a new bud. Some conidia of the mutant tng do not cease growth; instead, they continue to grow, sometimes reaching macroscopic sizes.

\section{CONIDIOPHORE TIMELINE}

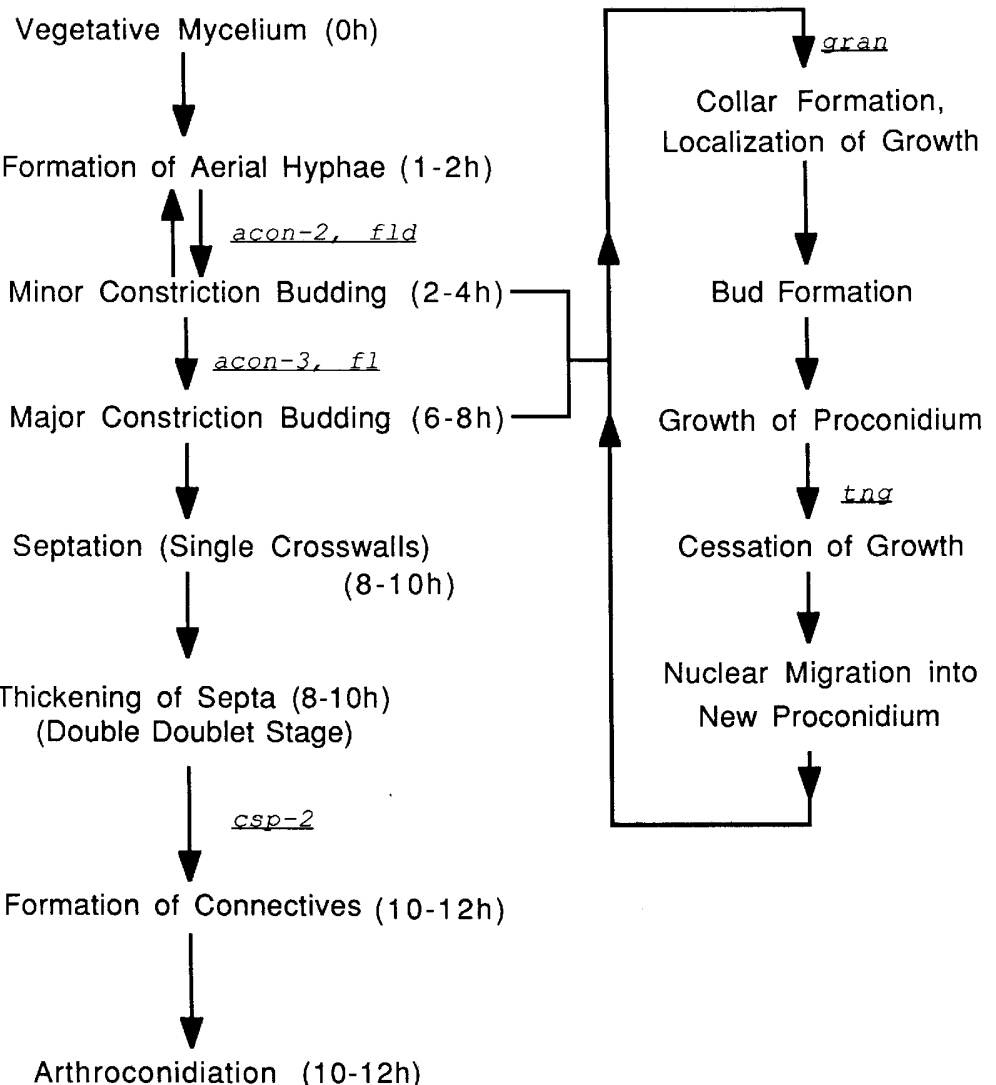

\section{BUDDING SUBROUTINE}

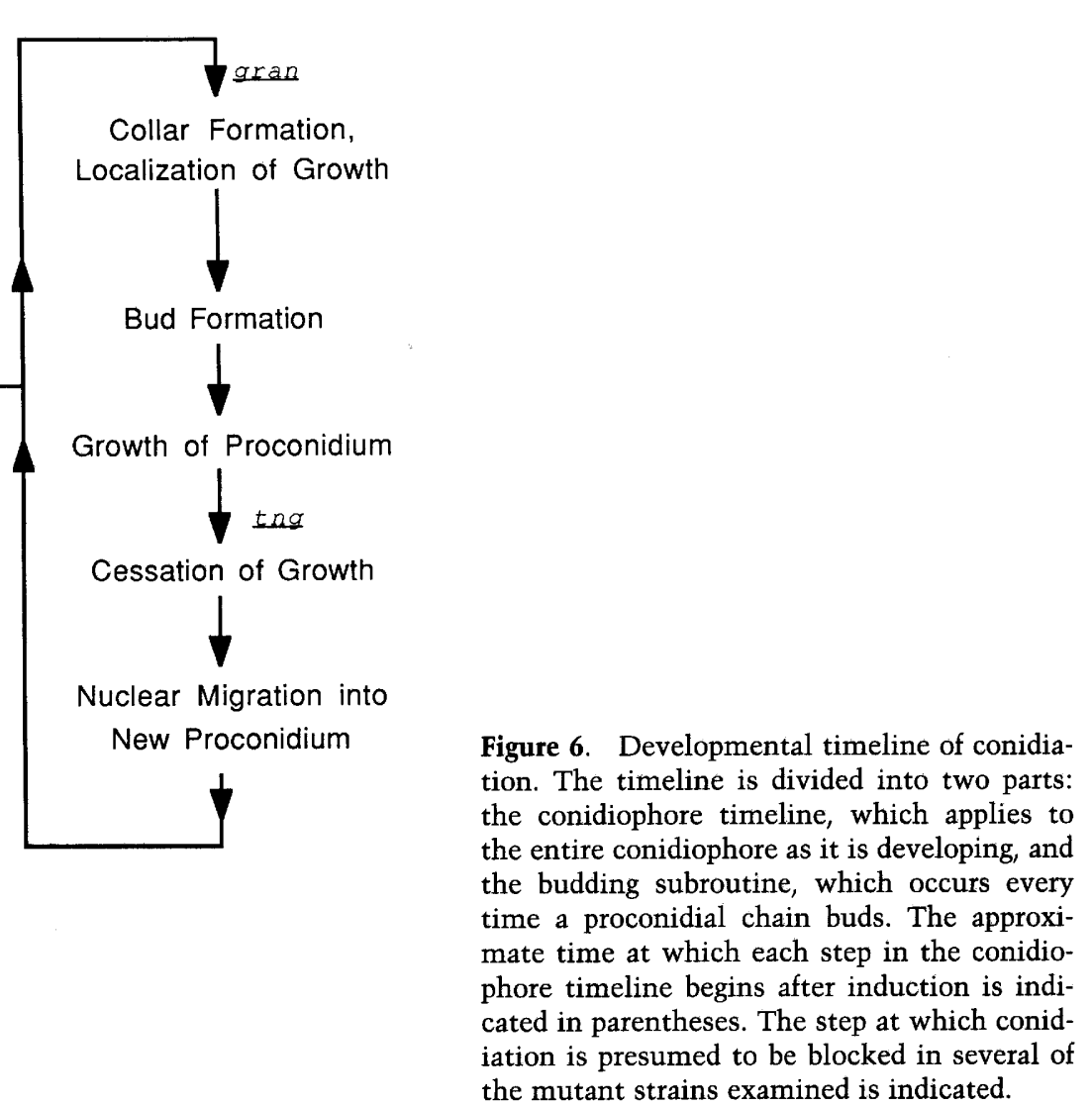


After a proconidial chain has finished growing, crosswalls form in the interconidial junctions. This heralds the beginning of the late events, in which the conidiophore matures and forms septa. When stained with Calcofluor, the cross-walls appear initially as thin single lines that thicken until they reach the stage descriptively referred to as the double-doublet stage. A furrow forms between adjacent conidia, which then separate, leaving a thin connective of Calcofluor-staining material holding them together. Conidia of the mutants csp-1, $c s p-2$, and $c y$ fail to separate but otherwise look normal. The nature of the csp-1 and cy defects is unclear, but csp-2 seems to be blocked at the double-doublet stage. Shortly after septation occurs, extra cross-walls appear in the hyphal regions adjacent to the conidiophore. These cross-walls undergo the same morphological changes that the interconidial septa do, resulting in the disarticulation of the hypha into arthroconidia.

\section{Morphological changes correspond to changes in gene expression}

Several genes that are expressed preferentially during conidiation (con genes) have been cloned (Berlin and Yanofsky 1985b). Transcriptional analysis has revealed that the aconidial mutants acon-2, acon-3, and $f l$, in which conidiation is blocked during the early events, fail to express some of these genes (Roberts and Yanofsky 1989). The mutants $c s p-1, c s p-2$, and eas, which are defective in the execution of the late events, express all of the cloned con genes. These results imply that expression of some of the con genes is dependent on an earlier event that is blocked in the aconidial mutants but occurs before the csp-1, csp-2, and eas blocks. Expression of the other con genes presumably occurs before the events that are blocked in the aconidial mutants.

Preliminary developmental Northern blot studies have shown that the genes not expressed in the aconidial mutants are turned on in wild type between 8 and $10 \mathrm{hr}$ into conidiation, a time corresponding to the onset of conidial septation and separation (M. Sachs, M. Springer, and C. Yanofsky, unpubl.). This finding demonstrates a striking correlation between the transition from early to late morphological events and the expression of a class of conidiation-specific genes.

\section{Minor constriction budding appears to mark $a$ developmental decision point}

Aerial growth in Neurospora fluctuates between hyphal tip elongation and minor constriction budding, but once major constriction budding is initiated, it continues until the conidiophore is completed. Thus, under normal environmental conditions, the minor constriction budding stage appears to be a developmentally uncommitted state, whereas the major constriction budding stage is committed to formation of mature conidia. However, germination of major constriction chain conidia marks a return to hyphal growth. The germination/conidiation cycle might be viewed as an oscillation between two states, a hyphal state and a major constric- tion conidial state, with a minor constriction stage in between. Three lines of evidence support this interpretation. (1) As discussed above, when the mode of growth changes from hyphal tip elongation to major constriction budding, it appears to pass through an indecisive minor constriction budding stage. (2) We have observed that under some conditions, the germ tubes of germinating conidia begin their growth in a manner reminiscent of minor constriction budding. Two or three minor constriction elements typically are formed, after which growth becomes strictly hyphal. (3) We have never seen a minor constriction germ tube arising from an arthroconidium, which has presumably never left the hyphal growth state. In this interpretation, the mode of growth must pass through the indecisive minor constriction stage to switch from hyphal growth to major constriction growth during conidiation, and it must pass through a brief and sometimes imperceptible version of minor constriction growth upon germination of major constriction chain conidia into growing hyphae.

\section{Budding and septation in N. crassa resembles that in yeast}

Budding and septation in N. crassa and Saccharomyces cerevisiae are strikingly similar. The events common to both organisms include budding of a vesicle that grows into a cell capable of budding again, retention of a small junction between the bud and the mother cell despite the growth of the bud, formation of a Calcofluorstaining septum at the junction, and subsequent separation at the junction leaving bud scars on one (yeast) or both ( $N$. crassa) cells (Cabib et al. 1973; Pringle et al. 1986). Thus, septation in yeast may provide a good model for septation in Neurospora conidiophores.

Actin has been visualized in situ in yeast by staining with fluorescently labeled phalloidins and often is co-localized with a chitin ring associated with septum formation (Kilmartin and Adams 1984). This suggests that actin filaments may act as a scaffold that organizes the assembly of chitin microfibrils into a septum, possibly corresponding to the highly ordered ring of membraneassociated filaments' observed in thin sections of yeast buds (Byers and Goetsch 1976). Actin can be visualized simultaneously as cortical spots and thin filaments running through the cell (Adams and Pringle 1984; Kilmartin and Adams 1984). The pattern is reminiscent of phalloidin staining of hyphae of Schizophyllum commune (Runeberg and Raudaskoski 1986) and Uromyces phaseoli (Hoch and Staples 1983). Because Neurospora contains actin (Allen and Sussman 1978; Sikora and Marzluf 1982), and septation in vegetative hyphae of the basidiomycete Trametes versicolor appears to be preceded by the formation of a ring of microfilaments (Girbardt 1979|, it is plausible to consider the possibility that actin plays a similar role in $N$. crassa septation.

The pattern of arthroconidiation suggests the existence of a conidial septation-inducing factor

Most of the conidia in a Neurospora culture are blastoconidia, which arise by successive apical budding. After 
interconidial septa form in the blastoconidiophore, extra septa appear in the hyphal region adjacent to the conidiophore, resulting in the ultimate disarticulation of the hypha into arthroconidia. The distance between extra cross-walls increases with increasing distance away from the blastoconidiophore. Mutants such as $a d h$ and $f r$, which make small and no blastoconidiophores, respectively, make more arthroconidia than wild type. These observations could be explained by the existence of one or more conidial septation-inducing factors, which would stimulate the formation, at interconidial junctions, of septa capable of thickening and undergoing separation. The activity of the factor would be induced during conidiation, which would be consistent with the fact that mutants that do not begin the conidiation process, such as acon-2, do not make arthroconidia. The factor would be produced in the blastoconidiophore or in its equivalent in mutants such as fr. Leakage or transport of the factor into the adjacent hyphal region would result in the formation of arthroconidial cross-walls. The apparent gradient of interseptal distance might correspond to a concentration gradient resulting from diffusion of the factor away from its site of synthesis. If this were the case, it would explain the higher incidence of extra cross-wall formation in the hyphal region nearest the conidiophore.

This model also could explain the increased production of arthroconidia in mutants such as $a d h$ and $f r$. These mutants may produce wild-type levels of factor despite the greatly reduced size of their blastoconidiophores. This would result in elevated levels of excess factor that could leak into the adjacent hyphal regions, resulting in greatly elevated septation and arthroconidiation relative to wild type.

\section{Materials and methods}

\section{Strains and culture conditions}

The strains used in this study were obtained from D.D. Perkins (Stanford University) and the Fungal Genetics Stock Center (University of Kansas Medical Center), and are listed in Table 1. Routine crosses and manipulations were performed as described by Davis and de Serres (1970) and Perkins (1986). Cultures were maintained on $1.5 \%$ agar slants with Vogel's minimal medium N (Vogel 1964).

\section{$S E M$}

Samples were inoculated onto spots of $2 \%$ agar containing Vogel's $\mathrm{N}$ medium in $60-\mathrm{mm}$ plastic petri dishes. The spots were made by pouring $\sim 1.5 \mathrm{ml}$ of lukewarm agar in such a way that it did not spread to the sides of the plate. In spot cultures, hyphae are able to spread beyond the edges of the agar without leaving the confines of the petri dish. The cultures were grown at $34^{\circ} \mathrm{C}$ until covered with hyphae and then at room temperature until maturity (usually one to two days). The dishes were flooded gently with fixative consisting of $2 \%$ gluteraldehyde and $1 \%$ paraformaldehyde in $0.1 \mathrm{M}$ potassium phosphate buffer $(\mathrm{pH} 7.2)$ and left for $2 \mathrm{hr}$ at room temperature. Samples were rinsed with buffer and then postfixed with $1 \% \mathrm{OsO}_{4}$ in buffer for $1 \mathrm{hr}$. After rinsing at least five times with distilled water, the samples were dehydrated stepwise by an ethanol series con- sisting of $30,50,70,95$, and $100 \%$ ethanol, 15 min per step. The samples were then treated with fresh $100 \%$ ethanol for $30 \mathrm{~min}$. The ethanol was replaced with hexamethyldisilazane (HMDS; Polysciences), which is a substitute for critical-point drying (Nation 1983), and left for $30 \mathrm{~min}$. The HMDS was pipetted off, and the samples were air-dried in the hood for $\sim 20 \mathrm{~min}$ or until completely dry. Blocks of samples including agar were cut away, mounted on metal stubs, sputter coated with $20 \mathrm{~nm}$ of gold, and viewed with a Philips 505 scanning electron microscope.

To observe the timing of developmental events, conidiation was induced by the method of Berlin and Yanofsky (1985a). Briefly, conidia were germinated at $1.5 \times 10^{6} / \mathrm{ml}$ in minimal medium in a round-bottom flask. Sterile air was bubbled through the liquid to aerate and agitate the culture. After $24 \mathrm{hr}$ at room temperature, the culture was filtered onto sterile filter paper. The paper and mycelium were placed on a layer of glass beads in a petri dish containing enough medium to keep the paper moist. The culture was left uncovered to grow in a sterile hood. At designated times, pieces of the mycelium were cut out, using sterilized bent surgical scissors, and pinned to dental wax that had been stuck to the bottom of small plastic dishes. The rest of the procedure was identical to that described above, except that the samples were transferred to new dishes without dental wax before the addition of HMDS, because HMDS dissolves dental wax.

\section{Fluorescent labeling}

Glass depression slides were autoclaved in glass petri dishes along with folded circles of filter paper. The depressions were filled to the top with Vogel's $\mathrm{N}$ medium supplemented with $1.5 \%$ agarose, which, unlike agar, does not autofluoresce. Samples were inoculated on the top of the agarose, the filter paper was moistened to prevent the agarose from dessicating, and the plates were incubated overnight at $34^{\circ} \mathrm{C}$. The following morning, the moist filters were removed to encourage conidiation, and the samples were kept at room temperature for several hours.

For cross-wall visualization, several drops of $10 \mu \mathrm{g} / \mathrm{ml}$ Calcofluor (Fluorescent Brightener 28, Sigma) were applied over the agarose, and a coverslip was gently put down over the drop gently so that the solution spread over the glass next to the depression. The best region of the sample for viewing was where the hyphae and conidiophores had grown out over the glass surface and were sandwiched thinly between the slide and the coverslip.

For nuclear staining, two methods were used. In the first method, a coverslip was taped down to the dry depression slide culture with two strips of double-stick tape along the long sides of the slide. The strips were cut thin enough to allow space over the glass surrounding the well. The sample was fixed by applying a drop of $70 \%$ ethanol to an open side of the slide. The ethanol was drawn underneath the coverslip by capillary action, and the sample was fixed for $10 \mathrm{~min}$. The sample was rinsed by adding a few drops of water to one side and withdrawing liquid from the other side with a piece of filter paper. The water was replaced in a similar fashion with a solution of $0.5 \mu \mathrm{g} / \mathrm{ml}$ Hoechst 33258 (Sigma) in $25 \%$ glycerol with $2 \% n$ propyl gallate to reduce fading (Giloh and Sedat 1982).

In the second method, the glass petri dishes that contained the slides were filled slowly with $70 \%$ ethanol, gently covering the cultures. The dishes were covered and left for $10 \mathrm{~min}$. Then the slides were removed from the dishes with forceps and allowed to air-dry. The Hoechst solution then was applied directly to the slide over the agar, and a coverslip was put down over the well so that the solution spread over the glass. 


\section{Fluorescence microscopy}

Samples were viewed with a Nikon Microphot FX epifluorescence microscope. Filter combinations for Calcofluor and Hoechst, respectively, were $380-425 \mathrm{~nm}$ excitation, $430-\mathrm{nm}$ dichroic mirror, and 450-nm barrier and 330-380 nm excitation, 400-nm dichroic mirror, and 420-nm barrier. For Calcofluor viewing, we used the violet filter combination instead of the UV filter combination normally used because it has the advantage of not causing the fluorescence to fade while still giving a bright picture. Photographs were taken with Kodak Ektachrome 400 ASA film.

\section{Acknowledgments}

We thank Karl Hager, Dorothy Newmeyer, David Perkins, Matthew Sachs, Chuck Staben, and Brian White for their critical comments on the manuscript. M.L.S. is indebted to Marc Orbach, David Perkins, Anne Roberts, Matthew Sachs, Chuck Staben, Tom Schmidhauser, and Fran Thomas for advice and helpful suggestions during the course of this study. This work was supported by U.S. Public Health Service grant AG-05568 from the National Institutes of Health. M.L.S. is a predoctoral trainee of the U.S. Public Health Service. C.Y. is a Career Investigator of the American Heart Association.

\section{References}

Adams, A.E.M. and J.R. Pringle. 1984. Relationship of actin and tubulin distribution to bud growth in wild-type and morphogenetic-mutant Saccharomyces cerevisiae. 1. Cell Biol. 98: 934-945.

Allen, E.D. and A.S. Sussman. 1978. Presence of an actin-like protein in mycelium of Neurospora crassa. J. Bacteriol. 135: $713-716$.

Beever, R.E. and G.P. Dempsey. 1978. Function of rodlets on the surface of fungal spores. Nature 272: 608-610.

Berlin, V. and C. Yanofsky. 1985a. Protein changes during the asexual cycle of Neurospora crassa. Mol. Cell. Biol. 5: 839848.

- 1985b. Isolation and characterization of genes differentially expressed during conidiation of Neurospora crassa. Mol. Cell. Biol. 5: 849-855.

Brody, S. 1981. Genetic and biochemical studies on Neurospora conidia germination and formation. In The fungal spore: Morphogenetic controls (ed. G. Turian and H.R. Hohl), pp. 605-626. Academic Press, New York.

Byers, B. and L. Goetsch. 1976. A highly ordered ring of membrane-associated filaments in budding yeast. J. Cell. Biol. 69: $717-721$.

Cabib, E., R. Ulane, and B. Bowers. 1973. A molecular model for morphogenesis: The primary septum of yeast. Curr. Topics Cell Regul. 8: 1-32.

Cole, G.T. 1986. Models of cell differentiation in conidial fungi. Microbiol. Rev. 50: 95-132.

Darken, M.A. 1961. Applications of fluorescent brighteners in biological techniques. Science 133: 1704-1705.

Davis, R.H. and F.J. de Serres. 1970. Genetic and microbiological research techniques for Neurospora crassa. Methods Enzymol. 17A: 79-143.

Giloh, H. and J.W. Sedat. 1982. Fluorescence microscopy: Reduced photobleaching of rhodamine and fluorescein protein conjugates by $n$-propyl gallate. Science 217: 1252-1255.
Girbardt, M. 1979. A microfilamentous septal belt (FSB) during induction of cytokinesis in Trametes versicolor (L. ex Fr.) Exp. Mycol. 3: 215-228.

Hoch, H.C. and R.C. Staples. 1983. Visualization of actin in situ by rhodamine-conjugated phalloin in the fungus Uromyces phaseoli. Eur. J. Cell Biol. 32: 52-58.

Hunsley, D. and G.W. Gooday. 1974. The structure and development of septa in Neurospora crassa. Protoplasma 82: $125-146$.

Kilmartin, J.V. and A.E.M. Adams. 1984. Structural rearrangements of tubulin and actin during the cell cycle of the yeast Saccharomyces. I. Cell Biol. 98: 922-933.

Maeda, H. and N. Ishida. 1967. Specificity of binding of hexopyranosyl polysaccharides with fluorescent brightener. $I$. Biochem. 62: 276-278.

Mahadevan, P.R. and E.L. Tatum. 1965. Relationship of the major constituents of the Neurospora crassa cell wall to wild-type and colonial morphology. J. Bacteriol. 90: 10731081.

Matsuyama, S.S., R.E. Nelson, and R.W. Siegel. 1974. Mutations specifically blocking differentiation of macroconidia in Neurospora crassa. Dev. Biol. 41: 278-287.

Nation, J.L. 1983. A new method using hexamethyldisilazane for preparation of soft insect tissues for scanning electron miscroscopy. Stain Technol. 58: 347-351.

Nelson, R.E., C.P. Selitrennikoff, and R.W. Siegel. 1975. Cell changes in Neurospora. In Results and problems in cell differentiation (ed. J. Reinert and H. Holzer), pp. 291-310. Springer-Verlag, Berlin.

Perkins, D.D. 1966. Details for collection of asci as unordered groups of eight projected ascospores. Neurospora Newsl. 9: 11 .

-1986. Hints and precautions for the care, feeding and breeding of Neurospora. Fungal Genetics Newsl. 33: 35-4l.

Perkins, D.D., A. Radford, D. Newmeyer, and M. Björkman. 1982. Chromosomal loci of Neurospora crassa. Microbiol. Rev. 46: 426-570.

Pringle, J.R., S.H. Lillie, A.E.M. Adams, C.W. Jacobs, B.K. Haarer, K.G. Coleman, J.S. Robinson, L. Bloom, and R.A. Preston. 1986. Cellular morphogenesis in the yeast cell cycle. In Yeast cell biology (ed. J. Hicks), pp. 47-80. Alan R. Liss, New York.

Roberts, A.N., V. Berlin, K.M. Hager, and C. Yanofsky. 1988. Molecular analysis of a Neurospora crassa gene expressed during conidiation. Mol. Cell. Biol. 6: 2411-2418.

Roberts, A.N. and C. Yanofsky. 1989. Genes expressed during conidiation in Neurospora Crassa: Characterization of con-8. Nucleic Acids Res. 17: 197-214.

Runeberg, P. and M. Raudaskoski. 1986. Cytoskeletal elements in the hyphae of the homobasidiomycete Schizophyllum commune visualized with indirect immunofluorescence and NBD-phallacidin. Eur. I. Cell. Biol. 41: 25-32.

Sargent, M.L. and S.H. Kaltenborn. 1972. Effects of medium composition and carbon dioxide on circadian conidiation in Neurospora. Plant Physiol. 50: 171-175.

Schmidt, J.C. and S. Brody. 1976. Biochemical genetics of Neurospora crassa conidial germination. Bacteriol. Rev. 40: $1-41$.

Scott, W.A. 1976. Adenosine $3^{\prime}: 5^{\prime}$-cyclic monophosphate deficiency in Neurospora crassa. Proc. Natl. Acad. Sci. 73: 2995-2999.

Seale, T. 1973. Life cycle of Neurospora crassa viewed by scanning electron microscopy. I. Bacteriol. 113: 1015-1025.

Selitrennikoff, C.P. 1976. Easily-wettable, a new mutant. Neurospora Newsl. 23: 23. 
Selitrennikoff, C.P., R.E. Nelson, and R.W. Siegel. 1974. Phasespecific genes for macroconidiation in Neurospora crassa. Genetics 78: 679-690.

Sikora, L. and G.A. Marzluff. 1982. Identification and isolation of actin from Neurospora crassa. J. Gen. Microbiol. 128: $439-445$.

Terenzi, H.F., M.M. Flawia, and H.N. Torres. 1974. A Neurospora crassa morphological mutant showing reduced adenylate cyclase activity. Biochem. Biophys. Res. Comm. 58: 990-996.

Timberlake, W.E. and M.A. Marshall. 1988. Genetic regulation of development in Aspergillus nidulans. Trends Genet. 4: $162-169$.

Turian, G. and N. Matikian. 1966. Conidiation of Neurospora crassa. Nature 212: 1067-1068.

Turian, G. and D.E. Bianchi. 1972. Conidiation in Neurospora. Bot. Rev. 38: 119-154.

Urey, J.C. 1971. Enzyme patterns and protein synthesis during synchronous conidiation in Neurospora crassa. Dev. Biol. 26: 17-27.

Vogel, H.J. 1964. Distribution of lysine pathways among fungi: Evolutionary implications. Am. Naturalist 98: 435-446. 


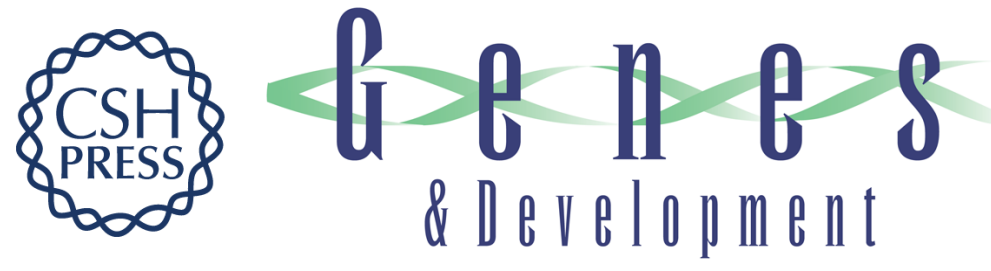

\section{A morphological and genetic analysis of conidiophore development in Neurospora crassa.}

M L Springer and C Yanofsky

Genes Dev. 1989, 3:

Access the most recent version at doi:10.1101/gad.3.4.559

References This article cites 38 articles, 16 of which can be accessed free at:

http://genesdev.cshlp.org/content/3/4/559.full.html\#ref-list-1

License

Email Alerting

Service

Receive free email alerts when new articles cite this article - sign up in the box at the top right corner of the article or click here.

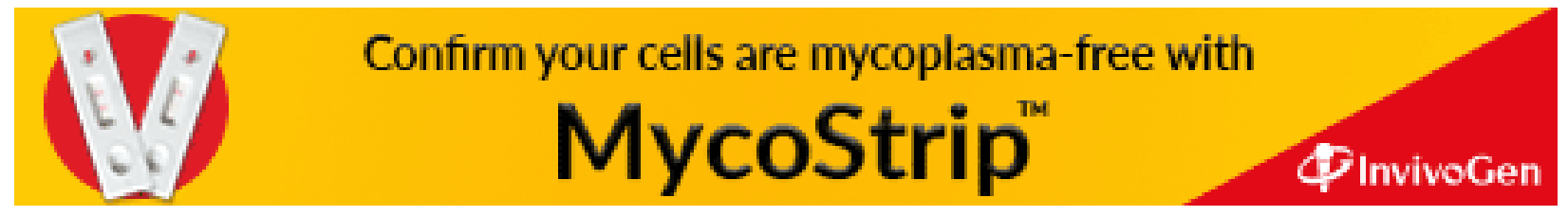

